Varia - Miscellaneous - Divers

Errata

\title{
On curvilinear distributions expressed by double Fourier series
}

\author{
By A. P. Zieliński, Dept. of Mechanics, Technical University of Cracow, Poland \\ Z. angew. Math. Phys. 31, 717-729 (1980)
}

By mistake figures 3 a) to c) and 4a) to d) on pages 727 and 728 have been crossed out. They are correct.

Buchbesprechungen - Book Reviews - Notices bibliographiques

Moderne mathematische Methoden in der Technik, Bd. 3. Von Stefan Fenyö (Birkhäuser Verlag, Basel-Boston-Stuttgart 1980) 348 S., 16 Fig.; sFr. 84,-/DM 92,-

Das Vorwort beginnt wie folgt: „Der vorliegende letzte Band unserer Serie ist den Grundbegriffen der Operatorentheorie und einiger seiner Anwendungen gewidmet. Die scheinbar sehr abstrakten Begriffe und Tatsachen der Funktionalanalysis spielen eine immer bedeutsamere Rolle in den verschiedenen technischen Disziplinen, unser Ziel ist mit diesem Band dem Praktiker die Aneignung des Stoffes durch eine praxisorientierte Darstellung zu erleichtern."

Das erste Kapitel (177 S.), ,Theorie der linearen Operatoren“, bringt eine mathematisch kompetente Einführung in die Funktionalanalysis. Vom didaktischen Standpunkt aus fraglich ist die Abwesenheit jeglicher Beispiele (auch solcher rein mathematischer Art) zu den zahlreichen Definitionen und Sätzen. Es braucht schon sehr brave Studenten, wenn sie während des Studiums dieses Teils den Glauben an die „Praxisnähe“ der Theorie nicht verlieren sollen. Kapitel 2, „Lineare Integralgleichungen", $(99 \mathrm{~S}$.) behandelt die Volterrasche und die Fredholmsche Gleichung, beides auch mit singulären Kernen. Es folgen selbstadjungierte Integraloperatoren nebst (kurz) den zugehörigen Entwicklungssätzen. Im dritten Kapitel (64 S.) folgen sodann ,Anwendungen der Integralgleichungen“; darunter fallen hier die Lösung von Randwertaufgaben bei gewöhnlichen Differentialgleichungen mit Hilfe der Greenschen Funktion sowie die Lösung von potentialtheoretischen Grundaufgaben mit Hilfe von Potentialen einer einfachen und einer Doppelschicht. Also alles wie in den guten alten Zeiten, als es noch keine Computer gab. P. Henrici

First order algebraic differential equations. Von Michihiko Matsuda, Lect. Notes in Maths. Bd. 804. (Springer-Verlag, Berlin-Heidelberg-New York 1980). 111 S.; DM 18,-

Es handelt sich nicht, wie der ahnungslose Leser des Titels glauben könnte, um die Lösung, oder auch nur um die qualitativen Eigenschaften der Lösungen, algebraischer Differentialgleichungen, sondern um die algebraische Theorie von Körpern, in denen eine „Ableitung " defíniert ist. Kennzeichnend sind etwa Sätze wie das nachstehende Theorem 11 (S. 70): „Let R be a differential algebraic function field of one variable over the coefficient field $K$. The number of those places in $\mathrm{R}$ such that their rings are not closed under the differentiation is finite." Der ahnungslose Leser wird sich fragen, und wird im dunkeln darüber gelassen, weshalb er sich für solche Dinge interessieren sollte. Aber der eingeweihte Leser weiß natürlich, daß es sich dabei um gute Mathematik handelt, die um ihrer innern Schönheit willen kultiviert wird und weil sie sich vielleicht später doch einmal als nützlich erweisen könnte.

P. Henrici

Vorlesungen über Approximation im Komplexen. Von Dieter Gaier, (Birkhäuser Verlag, Basel-Boston-Stuttgart 1980) 168 S.; sFr. 26,-/DM 28,--

Der Band gibt eine gründliche, knapp gefaßte und dabei doch angenehm lesbare Einführung in das aktuelle Gebiet der komplexen Approximationstheorie. Die Darstellung führt von den hi- 